\title{
The Human Skeletal Muscle Na Channel Mutation R669H Associated with Hypokalemic Periodic Paralysis Enhances Slow Inactivation
}

\author{
Arie F. Struyk, ${ }^{1}$ Kylie A. Scoggan, ${ }^{3}$ Dennis E. Bulman, ${ }^{3,4}$ and Stephen C. Cannon ${ }^{1,2}$ \\ Departments of ${ }^{1}$ Neurology, Massachusetts General Hospital, and ${ }^{2}$ Neurobiology, Harvard Medical School, Boston, \\ Massachusetts 02114, and ${ }^{3}$ Department of Medicine, Division of Neurology, and ${ }^{4}$ Ottawa Hospital Research Institute, \\ Ottawa, Ontario K1H 7W9 Canada
}

\begin{abstract}
Missense mutations of the human skeletal muscle voltage-gated $\mathrm{Na}$ channel (hSkM1) underlie a variety of diseases, including hyperkalemic periodic paralysis (HyperPP), paramyotonia congenita, and potassium-aggravated myotonia. Another disorder of sarcolemmal excitability, hypokalemic periodic paralysis $(\mathrm{Hy}-$ poPP), which is usually caused by missense mutations of the S4 voltage sensors of the L-type Ca channel, was associated recently in one family with a mutation in the outermost arginine of the IIS4 voltage sensor (R669H) of hSkM1 (Bulman et al., 1999). Intriguingly, an arginine-to-histidine mutation at the homologous position in the L-type $\mathrm{Ca}^{2+}$ channel $(\mathrm{R} 528 \mathrm{H})$ is a common cause of HypoPP. We have studied the gating properties of the hSkM1$\mathrm{R} 669 \mathrm{H}$ mutant $\mathrm{Na}$ channel experimentally in human embryonic
\end{abstract}

kidney cells and found that it has no significant effects on activation or fast inactivation but does cause an enhancement of slow inactivation. $\mathrm{R} 669 \mathrm{H}$ channels exhibit an $\sim 10 \mathrm{mV}$ hyperpolarized shift in the voltage dependence of slow inactivation and a twofold to fivefold prolongation of recovery after prolonged depolarization. In contrast, slow inactivation is often disrupted in HyperPP-associated $\mathrm{Na}$ channel mutants. These results demonstrate that, in R669H-associated HypoPP, enhanced slow inactivation does not preclude, and may contribute to, prolonged attacks of weakness and add support to previous evidence implicating the IIS4 voltage sensor in slow-inactivation gating.

Key words: depolarization; inactivation; Na channel; hypokalemic periodic paralysis; mutation; skeletal muscle
The human skeletal muscle voltage-gated sodium channel (hSkM1) mediates sarcolemmal excitability because of its rapid activation and subsequent fast inactivation in response to membrane depolarization. In addition to its fast gating, which takes place over milliseconds, the $\mathrm{Na}$ channel enters a more prolonged "slow"inactivated state, over hundreds of milliseconds to seconds, in response to sustained membrane depolarizations. The structural basis of $\mathrm{Na}$ channel slow inactivation is not known but appears to be different from conformational rearrangements associated with fast inactivation (Rudy, 1978; Cummins and Sigworth, 1996; Featherstone et al., 1996; Vedantham and Cannon, 1998). Slow inactivation may modulate $\mathrm{Na}$ channel availability, and thus sarcolemmal excitability, in response to prolonged shifts in membrane potential (Chandler and Meves, 1970; Almers et al., 1983; Ruff et al., 1988).

Gain-of-function mutations of the skeletal muscle $\mathrm{Na}$ channel gene SCN $4 A$ have been identified in a variety of disorders of muscle membrane excitability: potassium-aggravated myotonia, paramyotonia congenita (PMC), and hyperkalemic periodic paralysis (HyperPP) (for review, see Cannon, 2000). Attacks of weakness in HyperPP and PMC are characterized by a depolarizationinduced loss of membrane excitability. Depolarization arises from an aberrant inward Na current conducted by mutant channels with disrupted fast inactivation (Cannon et al., 1993; Hayward et al., 1996). Slow inactivation is impaired by three of the known HyperPP/PMC-linked mutations (Cummins and Sigworth, 1996; Hayward et al., 1997, 1999). This defect is thought to promote weakness by dampening the ability of the Na channels to turn off the persistent $\mathrm{Na}$ current and thereby to permit rapid (within

Received June 26, 2000; revised Sept. 11, 2000; accepted Sept. 15, 2000.

This work was supported by grants from the National Institutes of Health, the National Institute of Arthritis and Musculoskeletal and Skin Diseases Grant AR42703 to S.C.C. and the National Institute of Neurological Diseases and Stroke Grant K08-NS02137 to A.F.S., and by a grant from the Medical Research Council of Canada to D.E.B.

Correspondence should be addressed to Dr. Stephen C. Cannon, Department of Neurobiology, Massachusetts General Hospital, Edwards Research Building 417, Fruit Street, Boston, MA 02114. E-mail: cannon@helix.mgh.harvard.edu.

Copyright (C) 2000 Society for Neuroscience $0270-6474 / 00 / 208610-08 \$ 15.00 / 0$ seconds) repolarization and recovery of excitability (Ruff, 1994; Hayward et al., 1997, 1999).

Similar to HyperPP, hypokalemic periodic paralysis (HypoPP) is a rare autosomal-dominant disorder characterized by intermittent attacks of weakness, often lasting for hours to days. In HypoPP, however, weakness is accompanied by a decrease in serum potassium concentration, and myotonic stiffness does not occur (Rüdel et al., 1984; Cannon, 1998). During an attack, the sarcolemma becomes depolarized and inexcitable (Rüdel et al., 1984). The basis of this depolarization remains speculative, although changes in ATP-sensitive $\mathrm{K}$ channels have been promoted recently as direct precipitants (Ruff, 1999; Tricarico et al., 1999). Genetic analysis of HypoPP patients has identified three missense mutations in the $\alpha_{1}$ subunit of the skeletal muscle voltage-gated L-type calcium channel: R528H, R1239H, and R1239G (Fontaine et al., 1994; JurkatRott et al., 1994; Ptacek et al., 1994). These mutations disrupt the outermost arginine residues of the S4 membrane-spanning segments of either domain II or IV of the L-type Ca channel. A variety of gating defects have been reported for these mutants (Sipos et al., 1995; Lapie et al., 1996; Morrill et al., 1998; Morrill and Cannon, 1999), with little further insight into the pathophysiology of the disease.

Recently, two members of a French family were described with HypoPP, in which no L-type Ca channel mutation was identified. Single-strand conformational polymorphism analysis revealed an abnormal conformer of the Na channel gene $S C N 4 A$. A missense mutation that converts an arginine at position 669 to histidine $(\mathrm{R} 669 \mathrm{H})$ was identified that cosegregated with the disease phenotype (Bulman et al., 1999). This arginine is conserved in all voltage-gated $\mathrm{Na}$ channels, and the R669H mutation was not found as an incidental polymorphism in an analysis of 100 normal individuals. The $\mathrm{Na}$ channel $\mathrm{R} 669 \mathrm{H}$ mutation and the $\mathrm{Ca}$ channel $\mathrm{R} 528 \mathrm{H}$ mutation both replace the outermost arginine of the IIS4 voltage sensors of the respective channels by histidine.

We have studied the gating properties of the hSkM1-R669H mutant channels expressed heterologously in human embryonic kidney (HEK) cells. The kinetics and voltage dependence of acti- 
vation and fast inactivation were not significantly altered by this mutation. Surprisingly, slow inactivation was profoundly affected. In contrast to some HyperPP mutations, in which slow inactivation is disrupted, $\mathrm{R} 669 \mathrm{H}$ enhances slow inactivation, predominantly via prolonged recovery from slow inactivation and a hyperpolarized shift in the voltage dependence of slow inactivation. These results help to define the role of slow inactivation in disorders of muscle membrane excitability and support previous work implicating the IIS4 voltage sensor in slow-inactivation gating.

\section{MATERIALS AND METHODS}

Expression of sodium channels. cDNA encoding the adult isoform of the human muscle sodium channel $\alpha$ subunit hSkM1 (George et al., 1992) was used as a template for in vitro site-directed mutagenesis using the Transformer kit (Clontech, Palo Alto, CA). Primers CGTCGACGGATCGGGACATGTCCCGATCCCCTATGG and GACTGTCTGTCCTACACTCCTTCCG were used for selection and introduction of the missense mutation, respectively. In addition to introduction of the arginine-tohistidine missense codon, a translationally silent EclHK1 site was introduced into the cDNA-coding sequence by the mutant oligomer for the purpose of rapid screening. The human $\beta 1$ subunit cDNA was subcloned into the EcoRI site of the mammalian expression vector pcDNA1 (McClatchey et al., 1993). An independently derived hSkM1-R669H Na channel mutant clone (D. Bulman) was used in identical experimental protocols to confirm the results seen with our construct.

Cultures of HEK cells and their transient transfection were performed as described previously (Hayward et al., 1996). Briefly, supercoiled plasmid DNA encoding $1.25 \mu \mathrm{g}$ of either wild-type or $\mathrm{R} 669 \mathrm{H}$ mutant $\mathrm{Na}$ channel $\alpha$ subunits along with $2.5 \mu \mathrm{g}$ (fourfold molar excess) of the $\beta 1$ subunit expression plasmid and a CD8 marker were used to transfect HEK cell monolayers in $35 \mathrm{~mm}$ dishes by the calcium phosphate precipitation method. Two to three days after transfection, the HEK cells were briefly trypsinized and passaged to a $22 \mathrm{~mm}$ round glass coverslip for electrophysiological recording. Individual transfection-positive cells were identified by labeling with anti-CD8 antibodies cross-linked to microbeads (Dynal, Great Neck, NY) (Jurman et al., 1994).

Whole-cell recording. Na currents were measured by the use of conventional whole-cell recording techniques as described previously (Hayward et al., 1996). Recordings were made with an Axopatch 200A amplifier (Axon Instruments, Foster City, CA). The output was filtered at $10 \mathrm{kHz}$ and digitally sampled at $40 \mathrm{kHz}$ using an LM900 interface (Dagan, Minneapolis, MN). Data were stored to a 486-based computer using AxoBasic (Axon Instruments) data acquisition software. Patch electrodes were fabricated from borosilicate capillary tubes with a multistage puller (Sutter, Novato, CA). The shank of the pipette was coated with Sylgard, and the tip was heat-polished to a final tip resistance in the bath solution of $0.5-2.0$ $\mathrm{M} \Omega$. At least $80 \%$ of the series resistance was compensated by the analog circuitry of the amplifier, and the leakage conductance was corrected by digital scaling and subtraction of the passive current elicited by a $25 \mathrm{mV}$ depolarization from the holding potential. Cells with peak currents of $<1$ $\mathrm{nA}$ after step depolarization from -120 to $-10 \mathrm{mV}$ were excluded to reduce potential contamination by small endogenous $\mathrm{Na}$ currents occasionally observed in untransfected HEK cells. In addition, cells with peak currents $>20 \mathrm{nA}$ were excluded to reduce series resistance errors. Only cells with series resistances of $<5 \mathrm{M} \Omega$ were included in the data set. Individual cells were allowed to equilibrate for 7-10 min after achieving internal access before acquiring data. We occasionally observed cells with $\mathrm{Na}$ currents that differed from typical $\mathrm{Na}$ channel gating behavior, characterized by higher amplitude persistent $\mathrm{Na}$ current after a $10 \mathrm{msec}$ depolarization and a prolonged $\tau_{h}$. To reduce effects of these modal-gating shifts, data from these cells were not included in the final analysis.

For all experiments except those using a reversed $\mathrm{Na}$ gradient, the internal pipette solution contained (in mM): $105 \mathrm{CsF}, 35 \mathrm{NaCl}, 10 \mathrm{EGTA}$, and $10 \mathrm{HEPES}, \mathrm{pH} 7.4$ by $\mathrm{CsOH}$. Fluoride was used in the pipette solution to prolong seal stability for the purposes of slow-inactivation protocols. The bath contained (in $\mathrm{mM}$ ): $140 \mathrm{NaCl}, 4 \mathrm{KCl}, 2 \mathrm{CaCl}_{2}, 1 \mathrm{MgCl}_{2}, 5$ glucose, and $10 \mathrm{HEPES}, \mathrm{pH} 7.4$ by $\mathrm{NaOH}$. For the reversed $\mathrm{Na}$ gradient experiments, the internal pipette solution contained (in $\mathrm{mM}$ ): $130 \mathrm{NaCl}, 10$ HEPES, and 10 EGTA; the bath solution contained (in $\mathrm{mM}$ ): 150 choline$\mathrm{Cl}, 10 \mathrm{HEPES}, 2 \mathrm{CaCl}_{2}$, and $0.2 \mathrm{CdCl}_{2}$. All recordings were made at room temperature.

Data analysis. The data were analyzed and displayed by the use of a combination of computer programs: AxoBasic, Excel (Microsoft Corporation), and Origin (MicroCal). Conductance was calculated as:

$$
G(V)=I_{\text {peak }} /\left(V-E_{\text {rev }}\right),
$$

where the reversal potential $E_{\text {rey }}$ was measured experimentally for each cell. Steady-state fast and slow inactivation were fitted to a Boltzmann function with a nonzero pedestal, $I_{0}$, calculated as:

$$
I_{\text {test }} / I_{\max }=\left(I-I_{0}\right) /\left(1+\mathrm{e}^{\left(V-V_{1 / 2}\right) / k}\right)+I_{0},
$$

where $V_{1 / 2}$ is the half-maximum voltage and $k$ is the slope factor. The kinetics of fast inactivation was quantified from single-exponential fits to the macroscopic current decay and to the relaxation between a closed and inactivated state revealed by two-pulse protocols at voltages between -40 and $-60 \mathrm{mV}$. The time constant of the decay $\tau$ was estimated by fitting macroscopic Na currents $I$ to a single exponential plus a constant term, $I_{\infty}$, as:

$$
I=\left(I_{\max }-I_{\infty}\right) * \mathrm{e}^{-t / \tau}+I_{\infty},
$$

where $I_{\max }$ is the maximal amplitude and $t$ is the pulse duration.

The time course of recovery from fast inactivation was measured by the use of a two-pulse protocol. A $40 \mathrm{msec}$ conditioning pulse to $-10 \mathrm{mV}$ was applied to fast inactivate the channels fully, followed by a return to the recovery potential (between -120 and $-80 \mathrm{mV}$ ) for a variable interval. The fraction of available (recovered) channels was assayed with a test pulse to $-10 \mathrm{mV}$, and the time course of recovery was fit to the equation:

$$
I / I_{\text {ref }}=A *\left[1-\mathrm{e}^{-t / \tau}\right]
$$

$I$ is the peak amplitude of the current, which was normalized to the peak amplitude of a reference current, $I_{\text {ref }}$, elicited by a pulse to $-10 \mathrm{mV}$ before application of the conditioning pulse. $A$ is the maximal extent of recovery, $t$ is the recovery pulse duration, and $\tau$ is the time constant of recovery.

After prolonged depolarization, Na channel availability recovers with a complex time course characterized by multiple exponential components (Cummins and Sigworth, 1996). An "intermediate" component, $I_{M}$, recovers within 100-300 msec, slow-inactivated channels $\left(I_{S}\right)$ recover within 1-3 sec, and ultraslow-inactivated channels $\left(I_{U}\right)$ recover over minutes. Recovery from intermediate $\left(I_{M}\right)$ - and slow $\left(I_{S}\right)$-inactivated states was measured by the use of a two-pulse protocol with conditioning pulse lengths of up to $60 \mathrm{sec}$. Between trials, channels were allowed to recover fully by holding the membrane at $-120 \mathrm{mV}$ for a period of three times the conditioning pulse duration (up to $90 \mathrm{sec}$ ). For conditioning pulse lengths of $>60 \mathrm{sec}$, a sequential recovery protocol was used in which a single conditioning pulse was followed by a series of brief test pulses during the recovery interval. For the sequential recovery protocol, peak current from each test pulse was normalized to a reference current $\left(I_{\text {ref }}\right)$, measured as the mean peak value from four separate step depolarizations to $-10 \mathrm{mV}$ from the recovery potential, before the conditioning pulse. $I_{\text {test }} / I_{\text {ref }}$ values were then fit with a two-exponential decay function:

$$
I_{\text {test }} / I_{\text {ref }}=A_{1}\left(1-\mathrm{e}^{-t / \tau_{1}}\right)+A_{2}\left(1-\mathrm{e}^{-t / \tau_{2}}\right)+I_{0} .
$$

$A_{1}$ and $A_{2}$ are the amplitudes of the two components, $\tau_{1}$ and $\tau_{2}$ are the time constants, $t$ is the recovery time, and $I_{0}$ is the fractional current at time 0 . Data from cells whose current failed to recover within $10 \%$ of the reference current were discarded.

Symbols with error bars indicate means \pm SEM. Statistical significance was determined by the unpaired $t$ test with $p$ values noted in the text.

\section{RESULTS}

HyperPP-associated $\mathrm{Na}$ channel mutations have gain-of-function defects caused by disrupted fast inactivation or, in some cases, by a hyperpolarized shift in activation (Cannon, 2000). We sought to determine whether defects of fast gating, and in particular fast inactivation, might account for the HypoPP phenotype in our family. Wild-type and R669H mutant Na channel cDNAs under the control of a cytomegalovirus promoter were transiently transfected into HEK cells, along with the human isoform of the $\beta 1$ subunit. Whole-cell voltage-clamp recording was used to study the gating characteristics of the channels. On average, cells transfected with wild-type hSkM1 cDNA had slightly larger peak Na currents $(7.0 \pm 0.8 \mathrm{nA} ; n=36)$ than did those transfected with $\mathrm{R} 669 \mathrm{H}(5.6 \pm 0.4 \mathrm{nA} ; n=55)$, but the difference was not statistically significant $(p=0.10)$.

\section{Fast-gating behavior}

Representative current traces elicited by a series of step depolarizations from a holding potential of $-120 \mathrm{mV}$ to voltages ranging between -75 and $+80 \mathrm{mV}$ for wild-type (WT) and R669H channels are shown in Figure 1. Both the activation and inactivation properties of the $\mathrm{R} 669 \mathrm{H}$ current appeared to be qualitatively identical to that of wild type.

Three different protocols were used to quantify the kinetics of fast inactivation. The development of inactivation was measured over the voltage range -30 to $+40 \mathrm{mV}$ by fitting the current decay during a test depolarization. For membrane potentials between -60 and $-40 \mathrm{mV}$, the relaxation between closed and fastinactivated states was measured with a two-pulse protocol as the time-dependent inactivation of the peak current during variable duration conditioning pulses. Finally, the time constant of recovery from fast inactivation was determined by the use of a two-pulse 


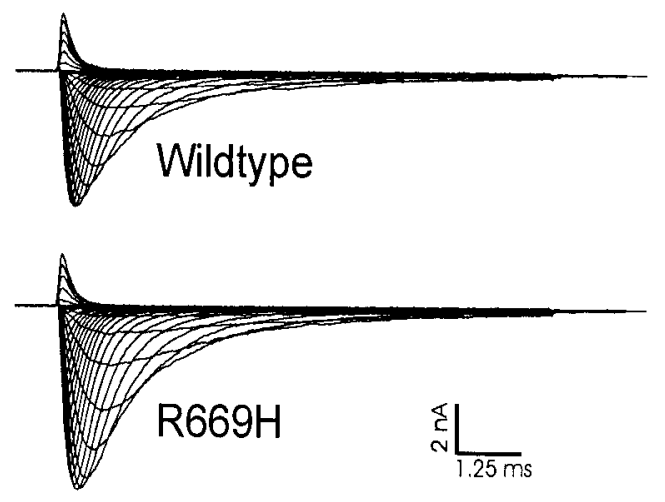

Figure 1. Representative currents from cells expressing WT or R669H channels. Currents were elicited by a series of voltage steps from a holding potential of $-120 \mathrm{mV}$ to voltages ranging between -75 and $+80 \mathrm{mV}$.
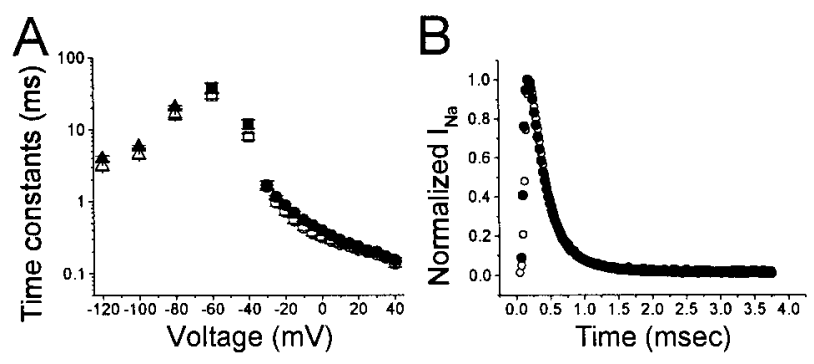

Figure 2. Fast-inactivation kinetics of R669H channels was indistinguishable from that of wild type. $A$, Time constants of fast inactivation at voltages between -30 and $+40 \mathrm{mV}$ for wild-type (open circles; $n=7$ ) and $\mathrm{R} 669 \mathrm{H}$ (closed circles; $n=10$ ) channels were determined by fitting a single exponential to the macroscopic current decay during step depolarizations from a holding potential of $-120 \mathrm{mV}$. At more hyperpolarized potentials $(-40$ and $-60 \mathrm{mV}$ ), a two-pulse protocol was used to measure the time course of fast inactivation from closed states for wild-type (open squares; $n=6$ ) and $\mathrm{R} 669 \mathrm{H}$ (closed squares; $n=7$ ) channels. The kinetics of recovery from fast inactivation for wild-type (open triangles; $n=6$ ) and $\mathrm{R} 669 \mathrm{H}$ (closed triangles; $n=6$ ) channels was determined by the use of a standard two-pulse protocol with a $40 \mathrm{msec}$ conditioning pulse at $-10 \mathrm{mV}$ and recovery potentials of $-80,-100$, or $-120 \mathrm{mV}$. $B$, The limiting rate of macroscopic inactivation at strongly depolarized potentials was identical for WT (open circles) and $\mathrm{R} 669 \mathrm{H}$ (closed circles) channels. Superimposed traces show outward $\mathrm{Na}^{+}$currents recorded with a reversed $\mathrm{Na}^{+}$gradient (see Materials and Methods) by depolarization to $+50 \mathrm{mV}$ from a holding potential of $-120 \mathrm{mV}$. The mean time constant of current decay was identical for wild-type $(0.26 \pm 0.008 \mathrm{msec} ; n=3)$ and $\mathrm{R} 669 \mathrm{H}(0.26 \pm 0.007 \mathrm{msec} ; n=$ 4) channels.

protocol with a variable recovery gap between the conditioning and test pulses. Recovery was measured at potentials ranging between -120 and $-80 \mathrm{mV}$. Figure $2 A$ displays the voltage dependence of the resulting time constants on a semilogarithmic scale. Time constants for both entry and recovery were comparable for $\mathrm{R} 669 \mathrm{H}$ channels and wild-type ones. The small increase in $\tau$ observed for $\mathrm{R} 669 \mathrm{H}$ was not statistically different from that of WT. The transition rate from open to the fast-inactivated state is estimated best from the asymptotic value of $\tau$ at strongly depolarized potentials. We examined this microscopic inactivation rate in greater detail by using a reversed $\mathrm{Na}$ gradient (high internal/low external) to shift $E_{\text {rev }}$ away from $+50 \mathrm{mV}$ and thereby increase the amplitude of the Na current. Superposition of individual trials showed no apparent differences between R669H and WT (Fig. 2B). On average, the single-exponential fit of the current decay elicited by a step depolarization from -120 to $+50 \mathrm{mV}$ yielded identical fast-inactivation time constants for wild-type $(0.26 \pm 0.008 \mathrm{msec} ; n=3)$ and $\mathrm{R} 669 \mathrm{H}$ $(0.26 \pm 0.007 \mathrm{msec} ; n=4)$ channels.

The peak conductance-voltage relationship of $\mathrm{R} 669 \mathrm{H}$ channels (Fig. 3) had an increased slope and a very small rightward shift, estimated to be a $3 \mathrm{mV}$ depolarized shift in the midpoint of the fitted curve in comparison with that of wild type (Table 1). The voltage dependence of steady-state fast inactivation was measured

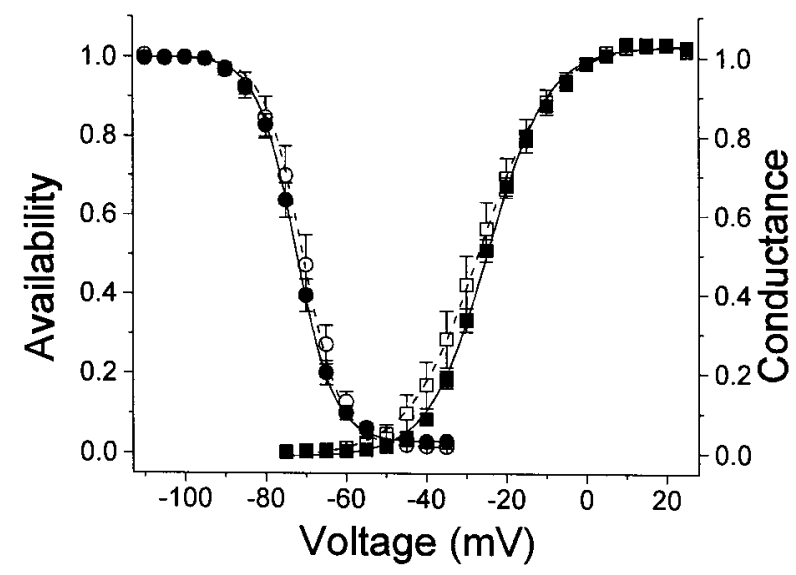

Figure 3. Voltage dependence of fast gating was not affected by the R669H mutation. The voltage dependence of fast inactivation of wild-type (open circles; $n=8$ ) and $\mathrm{R} 669 \mathrm{H}$ (closed circles; $n=11$ ) channels was measured as the peak $I_{\mathrm{Na}}$ elicited by a test depolarization to $-10 \mathrm{mV}$, after $300 \mathrm{msec}$ conditioning pulses to potentials ranging from -140 to $-35 \mathrm{mV}$. Fitted curves are derived from mean parameter values of $\chi^{2}$-minimized fits to a Boltzmann function plus a constant for each cell. The conductance-voltage curves for wild-type (open squares; $n=8$ ) and $\mathrm{R} 669 \mathrm{H}$ (closed squares; $n=$ 16) channels were derived from the peak $I_{\mathrm{Na}}$ elicited by a series of $10 \mathrm{msec}$ test pulses to the potentials ranging from -75 to $+80 \mathrm{mV}$. Reversal potential was fitted as an independent parameter for each cell. Curves for wild type and $\mathrm{R} 669 \mathrm{H}$ are computed from means of parameter values estimated from $\chi^{2}$-minimized fits of a Boltzmann function for each cell. Mean parameter values and SEMs are listed in Table 1.

as the relative peak current elicited after a $300 \mathrm{msec}$ conditioning pulse. Data from each cell were fit with a Boltzmann function, and amplitude-normalized data from separate cells were pooled as shown by mean values and SEMs in Figure 3. No significant difference was noted for the midpoint or the voltage sensitivity between $\mathrm{R} 669 \mathrm{H}$ and wild-type channels (Table 1).

\section{Slow inactivation}

Studies of HyperPP mutants suggest that, whereas defects in fast gating may precipitate episodes of paralysis, disruption of $\mathrm{Na}$ channel slow inactivation may contribute to the maintained depolarization responsible for prolonged attacks (Hayward et al., 1999). We therefore sought to characterize slow inactivation of the R669H mutant.

The rate of entry to the slow-inactivated state was measured by the use of a two-pulse protocol as described in the Figure $4 \mathrm{~A}$ inset. Cells were held at $-120 \mathrm{mV}$, and a conditioning pulse of varying duration was applied. A $20 \mathrm{msec}$ return to $V_{\text {hold }}$ was interposed between the conditioning and test pulses, which allowed complete recovery from fast inactivation. The current elicited by a subsequent pulse to $-10 \mathrm{mV}$ was normalized by the response to a preconditioning reference pulse, plotted against the conditioning pulse duration, and fit with a single-exponential function. The rate of entry to slow inactivation was similar for wild-type and $\mathrm{R} 669 \mathrm{H}$ channels at voltages between -80 and $-10 \mathrm{mV}$ (Fig. 4). The extent of slow inactivation, however, was greater for $\mathrm{R} 669 \mathrm{H}$ as shown by the lower relative $I_{\mathrm{Na}}$ after long conditioning pulses.

The voltage dependence of slow inactivation was determined by using a $40 \mathrm{sec}$ conditioning pulse (Fig. 5, inset), which was sufficiently long to allow slow inactivation to approach steady state, as shown in Figure 4. A $20 \mathrm{msec}$ gap at $-120 \mathrm{mV}$ was used to allow channels to recover from fast inactivation, and the fraction of channels not slow inactivated was measured as the relative peak current elicited by a subsequent test depolarization to $-10 \mathrm{mV}$. Slow inactivation was enhanced by $\mathrm{R} 669 \mathrm{H}$. The steady-state voltage dependence for $\mathrm{R} 669 \mathrm{H}$ was shifted by $10 \mathrm{mV}$ in the hyperpolarized direction compared with wild-type channels (Table 2), and the maximal extent of slow inactivation at strongly depolarized potentials was greater (Fig. 5, smaller relative current,).

With prolonged depolarization, $\mathrm{Na}$ channel inactivation is a 
Table 1. Voltage dependence of fast-gating transitions ${ }^{a}$

\begin{tabular}{|c|c|c|c|c|c|c|}
\hline & \multicolumn{3}{|l|}{ Activation } & \multicolumn{3}{|l|}{ Inactivation } \\
\hline & $V_{1 / 2}(\mathrm{mV})$ & $k(\mathrm{mV})$ & $n$ & $V_{1 / 2}(\mathrm{mV})$ & $k(\mathrm{mV})$ & $n$ \\
\hline WT & $-27.0 \pm 2.5$ & $8.0 \pm 0.2$ & 8 & $-70.9 \pm 1.6$ & $4.7 \pm 0.1$ & 8 \\
\hline $\mathrm{R} 669 \mathrm{H}$ & $-24.5 \pm 0.8$ & $7.2 \pm 0.3$ & 16 & $-72.5 \pm 0.2$ & $4.7 \pm 0.2$ & 11 \\
\hline
\end{tabular}

${ }^{a}$ The differences in the mean values were not different from $0(p>0.05)$ for any of the estimated gating parameters.

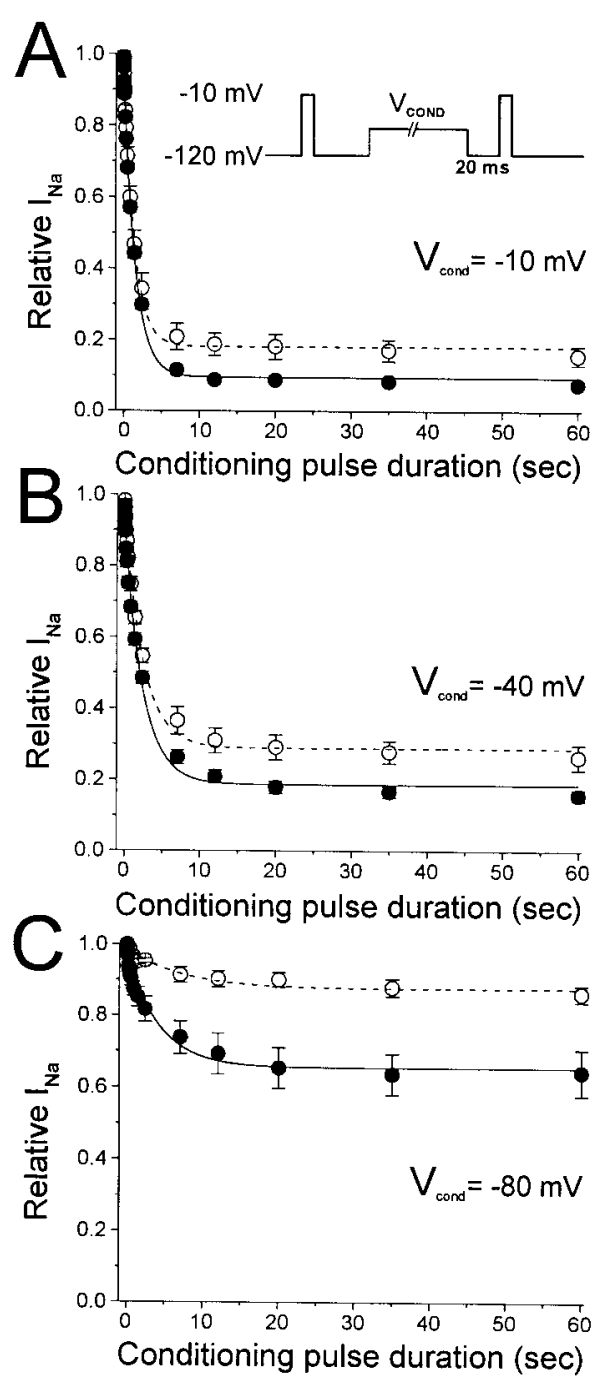

Figure 4. Entry to slow inactivation was enhanced for R669H channels. Entry to slow inactivation was measured as the peak $I_{\mathrm{Na}}$ elicited by a test depolarization to $-10 \mathrm{mV}$, after a conditioning pulse of varying duration. Current amplitudes were normalized to a reference peak, $I_{\mathrm{Na}}$, elicited by a depolarization to $-10 \mathrm{mV}$ from a holding potential of $-120 \mathrm{mV}$. Conditioning and test pulses were separated by a $20 \mathrm{msec}$ repolarization to -120 $\mathrm{mV}$ to allow for full recovery from fast inactivation $(A$, inset, pulse protocol). Data for wild type (open circles; $n=4$ ) and R669H (closed circles; $n=$ 9) were fit to a single-exponential relaxation to a steady-state value, $S_{\infty}$. Curves were generated from means of parameter values. $A, V_{\text {cond }}=-10$ $\mathrm{mV} ; \mathrm{WT}, \tau=390 \pm 140 \mathrm{msec}, S_{\infty}=0.18 \pm 0.03 ; \mathrm{R} 669 \mathrm{H}, \tau=510 \pm 74$ $\mathrm{msec}, S_{\infty}=0.09 \pm 0.01 . B, V_{\text {cond }}=-40 \mathrm{mV} ; \overline{\mathrm{WT}}, \tau=2400 \pm 60 \mathrm{msec}, \bar{S}_{\infty}=$ $0.29 \pm 0.03 ; \mathrm{R} 669 \mathrm{H}, \tau=2400 \pm 120 \mathrm{msec}, S_{\infty}=0.18 \pm 0.01 . C, V_{\text {cond }}=-80$ $\mathrm{mV}$; WT, $\tau=7100 \pm 2200 \mathrm{msec}, S_{\infty}=0.88 \pm 0.03 ; \mathrm{R} 669 \mathrm{H}, \tau=4200 \pm 800$ $\mathrm{msec}, S_{\infty}=0.65 \pm 0.05$.

complex process with multiple kinetically defined states with different entry and recovery rates (Cummins and Sigworth, 1996). Variations in the length of the conditioning pulse alter the population of these states, as evidenced by changes in the relative amplitudes of the multiexponential relaxation components (Hayward et al., 1997). We designed protocols to examine the recovery

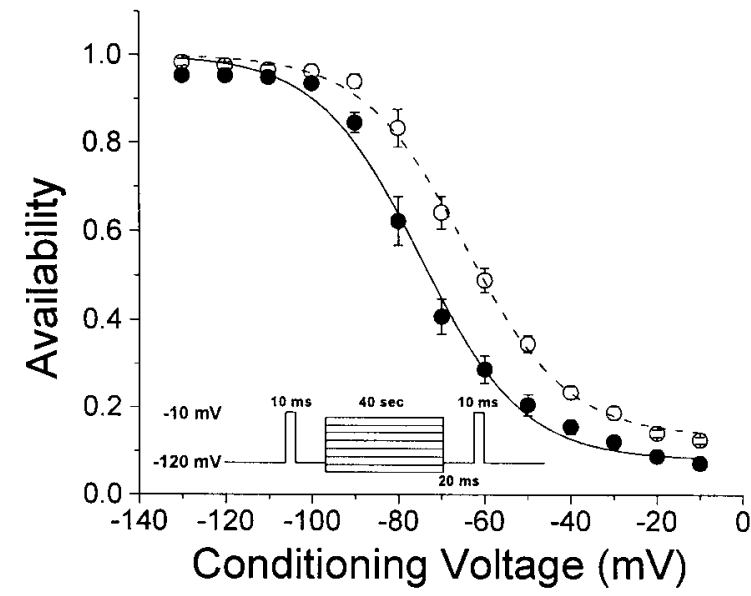

Figure 5. Steady-state slow inactivation was enhanced for $\mathrm{R} 669 \mathrm{H}$ channels. The voltage dependence of slow inactivation was measured as the peak $I_{\mathrm{Na}}$ elicited by a test depolarization to $-10 \mathrm{mV}$, after a $40 \mathrm{sec}$ conditioning pulse to voltages ranging from -130 to $-10 \mathrm{mV}$ for wild type (open circles $n=7$ ) and $\mathrm{R} 669 \mathrm{H}$ (closed circles; $n=10$ ). Current amplitudes were normalized to a reference peak, $I_{\mathrm{Na}}$, elicited by a depolarization to $-10 \mathrm{mV}$ from a holding potential of $-120 \mathrm{mV}$. Conditioning and test pulses were separated by a $20 \mathrm{msec}$ repolarization to $-120 \mathrm{mV}$ to allow recovery from fast inactivation (inset, pulse protocol). Smooth curves for wild-type data (dashed line) and $\mathrm{R} 669 \mathrm{H}$ (solid line) are computed from means of parameter values estimated from $\chi^{2}$-minimized fits to a Boltzmann function plus a constant term for each cell. Parameter values are listed in Table 2.

Table 2. Voltage dependence of slow inactivation

\begin{tabular}{llllr} 
& $V_{1 / 2}(\mathrm{mV})$ & $k(\mathrm{mV})$ & $S_{\mathrm{o}}$ & $n$ \\
\hline WT & $-64.5 \pm 1.7$ & $11.9 \pm 0.7$ & $0.13 \pm 0.02$ & 7 \\
R669H & $-74.8 \pm 2.1^{*}$ & $11.9 \pm 0.6$ & $0.08 \pm 0.01^{*}$ & 10 \\
\hline
\end{tabular}

*p $p<0.01$.

of channel availability after a wide range of conditioning pulse durations. Two different pulse protocols were used: two pulse and sequential. The two-pulse protocol was used for conditioning pulses of durations between $40 \mathrm{msec}$ and $60 \mathrm{sec}$ (Fig. 6, inset ). Full recovery between trials was verified by monitoring the current elicited by a reference pulse to $-10 \mathrm{mV}$ that preceded the conditioning pulse. The recovery interval between trials was generally three times the duration of the conditioning pulse, and peak current during the reference pulse did not vary by $>5 \%$. Recovery curves from $40 \mathrm{msec}$ conditioning pulses for both wild-type and R669H channels were well fit with a single exponential (Fig. 6A), consistent with the data from Figure 4 suggesting that a negligible fraction of the channels enter a slow-inactivated state within that time. The time constant for recovery from fast inactivation at -120 $\mathrm{mV}$ was similar for WT $(3.2 \pm 0.49 \mathrm{msec} ; n=6)$ and $\mathrm{R} 669 \mathrm{H}(4.0 \pm$ $0.38 \mathrm{msec} ; n=13$ ) channels. For conditioning pulses of $1 \mathrm{sec}$ or longer (Fig. 6B,C), however, a double-exponential relaxation function was required to obtain a reasonable fit. After a 1 sec conditioning pulse (Fig. $6 \mathrm{~B}$ ), $\sim 60 \%$ of WT and R669H channels recovered within $10 \mathrm{msec}$, and we interpret this as the fraction of channels that were fast inactivated. The second component recovered more slowly for R669H (530 $\pm 65 \mathrm{msec} ; n=9)$ than for WT 


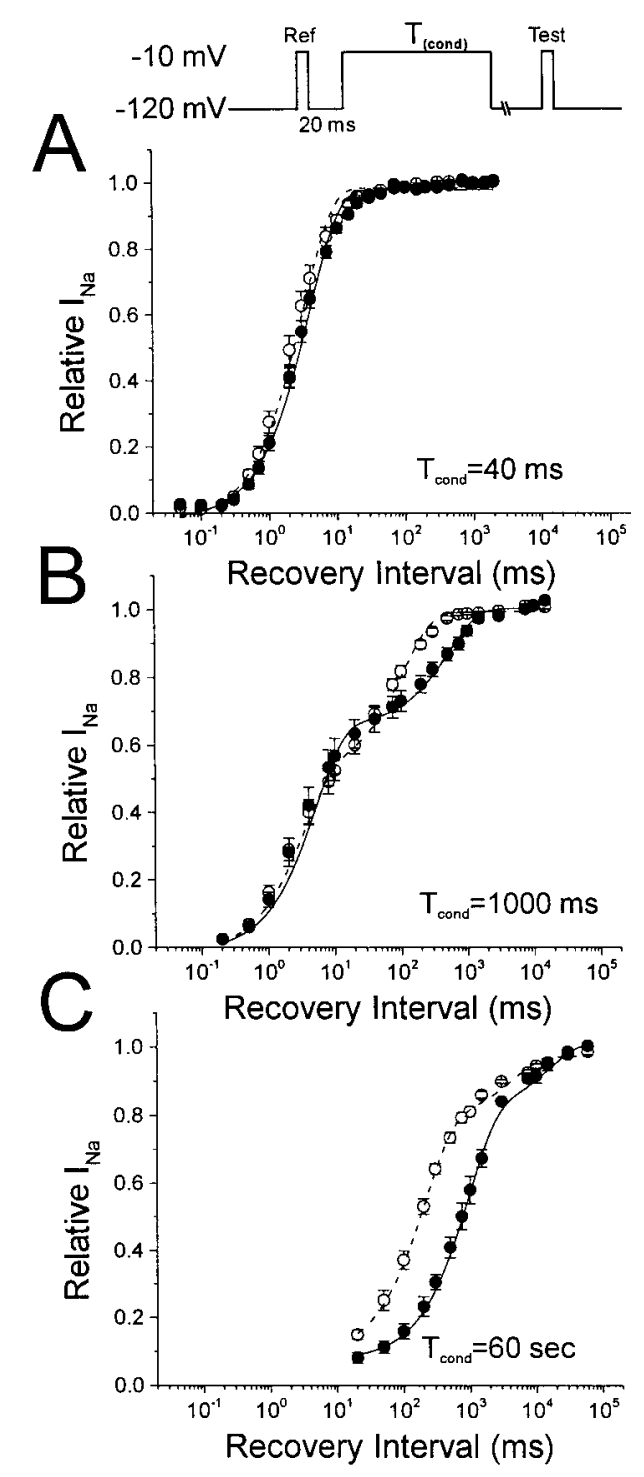

Figure 6. Recovery from an intermediate inactivation state $\left(I_{M}\right)$ was prolonged for $\mathrm{R} 669 \mathrm{H}$ channels. In $A-C$, a two-pulse protocol (inset) was used to measure recovery from inactivation for wild-type (open circles) and $\mathrm{R} 669 \mathrm{H}$ (closed circles) channels. The relative amplitude of different kinetic components of recovery varied with the duration of the conditioning pulse to $-10 \mathrm{mV}$ of $40 \mathrm{msec}(A), 1 \mathrm{sec}(B)$, or $60 \mathrm{sec}(C)$. The time course of recovery was measured as the relative peak $I_{\mathrm{Na}}$ at $-10 \mathrm{mV}$, as a function of the recovery interval at $-120 \mathrm{mV}$. Channels were allowed to recover fully at $-120 \mathrm{mV}$ between trials, as ascertained by the reference peak $I_{\mathrm{Na}}$ measured by a pulse to $-10 \mathrm{mV}$ before application of the conditioning pulse. The amplitude of this $I_{\text {ref }}$ was used to compute the relative degree of recovery for the subsequent test pulse to $-10 \mathrm{mV}$. A, After a $40 \mathrm{msec}$ conditioning pulse, recovery was well fit by a single exponential that was comparable for WT $(3.2 \pm 0.49 \mathrm{msec} ; n=6)$ and R669 (4.0 $\pm 0.38 \mathrm{msec}$; $n=13$ ) channels. $B$, Recovery after a 1 sec conditioning pulse had two exponential components. The fast component was similar for WT and $\mathrm{R} 669 \mathrm{H}$ channels, whereas the slower one $\left(I_{M}\right)$ was clearly prolonged for $\mathrm{R} 669 \mathrm{H}(530 \pm 65 \mathrm{msec} ; n=9)$ compared with WT $(110 \pm 12 \mathrm{msec} ; n=11)$. $C$, After a $60 \mathrm{sec}$ conditioning pulse, the time course of recovery had three components: fast ( $<10 \mathrm{msec} ; \sim 15 \%$ of total; not resolved by this protocol), intermediate $(100-500 \mathrm{msec} ; \sim 70 \%$ of response), and slow (5-15 sec; $\sim 15 \%$ of response). A double-exponential fit of the data was used to estimate the time constants of the intermediate and slow components. As in $B$, recovery of the intermediate component was markedly slower for $\mathrm{R} 669 \mathrm{H}$ than for WT, and recovery of the slow component was moderately prolonged $[16 \pm 5.5 \mathrm{sec}(n=5)$ for $\mathrm{R} 669 \mathrm{H}$ compared with $4.4 \pm 0.86 \mathrm{sec}(n=$ 6) for $\mathrm{WT}]$.

$(110 \pm 12 \mathrm{msec} ; n=11)$ channels. This component of recovery (typically $100-300 \mathrm{msec}$ at $-120 \mathrm{mV}$ ) is thought to reflect a population of channels that is in an intermediate-inactivated state, designated $I_{M}$, and the data in Figure $6, B$ and $C$, show that

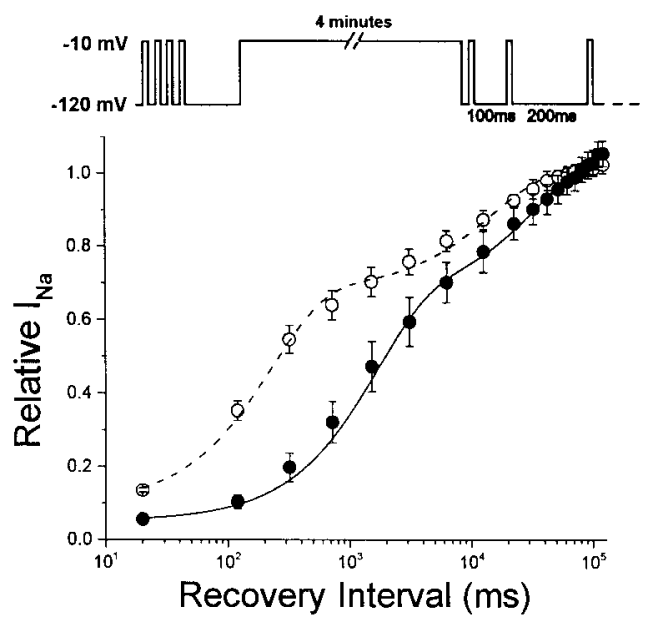

Figure 7. Recovery after long-duration conditioning pulses was prolonged for $\mathrm{R} 669 \mathrm{H}$ mutants. A $240 \mathrm{sec}$ conditioning pulse to $-10 \mathrm{mV}$ was applied from a resting potential of $-120 \mathrm{mV}$. Recovery from inactivation for wild-type (open circles) and R669H (closed circles) channels was measured by determining peak $I_{\mathrm{Na}}$ during $5 \mathrm{msec}$ test depolarizations to $-10 \mathrm{mV}$ from the recovery potential of $-120 \mathrm{mV}$ at the times indicated. Peak amplitudes were normalized to a reference response obtained as the mean of four separate pulses to $-10 \mathrm{mV}$ elicited before the conditioning pulse (see inset, pulse protocol). Because relaxation from the various inactivated states varied over five orders of magnitude, a logarithmic scale is used to show the time course of recovery. Recovery of the intermediate component was sevenfold slower for R669H than for WT, whereas the slow component was prolonged only approximately twofold. Smooth curves for wild type (dashed line) and R669H (solid line) were generated using means of parameter values estimated from [chi ${ }^{2}$-minimized fits of a double-exponential decay function plus a constant, $S_{\infty}$, for each cell. The two exponentials represent recovery of the intermediate- and slow-inactivated components, and the constant term is the proportion of channels that fully recovered within $20 \mathrm{msec}$ at $-120 \mathrm{mV}$. The relative amplitudes and time constants were as follows: WT, $S_{\infty}=0.091 \pm 0.007, \mathrm{~A}_{1}=0.59 \pm 0.04, \tau_{1}=230 \pm 21$ msec, $\mathrm{A}_{2}=0.33 \pm 0.02, \tau_{2}=15000 \pm 720 \mathrm{msec}, n=7 ; \mathrm{R} 669 \mathrm{H}, S_{\infty}=$ $0.051 \pm 0.007, \mathrm{~A}_{1}=0.61 \pm 0.05, \tau_{1}=1600 \pm 220 \mathrm{msec}, \mathrm{A}_{2}=0.41 \pm 0.04$, $\tau_{2}=37000 \pm 4000 \mathrm{msec}, n=11$.

recovery from $I_{M}$ was prolonged by $\mathrm{R} 669 \mathrm{H}$. The time course of recovery after a $60 \mathrm{sec}$ conditioning pulse (Fig. $6 \mathrm{C}$ ) was also well fit by a two-exponential relaxation, plus a small offset to account for the small fraction of channels $(\sim 10-15 \%)$ that recovered before the first time point at 10 msec. The bulk of WT and R669H channels recovered with the kinetics of $I_{M}$, and like the data after a $1 \mathrm{sec}$ conditioning pulse (Fig. $6 B$ ), recovery of this component was impeded for R669H $(930 \pm 140 \mathrm{msec})$ compared with WT $(200 \pm 18 \mathrm{msec})$. The second exponential component in Figure $6 C$ recovered on the order of seconds and reflects the fraction of slow-inactivated channels $\left(I_{S}\right)$. Recovery of the $I_{S}$ component is also prolonged by $\mathrm{R} 669 \mathrm{H}(16 \pm 5.5 \mathrm{sec} ; n=5)$ compared with WT $(4.4 \pm 0.86 \mathrm{sec} ; n=6)$. The estimate for the time constant of the $I_{S}$ component is limited by its small amplitude, $\sim 10 \%$ of the total current. In the next set of experiments even longer duration conditioning pulses of several minutes were used to obtain a better measure of the rate of recovery for the $I_{S}$ component.

Because conditioning pulse durations $>1$ min and their associated slow recovery times are impractical for two-pulse protocols, a sequential protocol with a single conditioning pulse followed by a series of short test depolarizations was used to monitor the time course of recovery after such prolonged conditioning pulses (Fig. 7). The brief and relatively infrequent test depolarizations do not alter the recovery from $I_{M}$ and slower components, as judged by pilot studies showing indistinguishable recovery curves using conventional two-pulse or this sequential protocol (data not shown). The peak $I_{\mathrm{Na}}$ elicited from these test depolarizations was normalized to the reference "maximal" $I_{\mathrm{Na}}$ measured as the average response from four step depolarizations preceding the conditioning pulse. After a $240 \mathrm{sec}$ conditioning pulse, $\sim 60 \%$ of the channels recovered with a time course typical of $I_{M}, 35 \%$ recovered with the 
kinetics of $I_{S}$, and $\sim 5 \%$ of the channels were fast inactivated only (Fig. 7). As shown by the first data point in the recovery curve, the fraction of channels fast inactivated only was smaller for $\mathrm{R} 669 \mathrm{H}$ $(0.05 \pm 0.01 ; n=11)$ compared with WT $(0.09 \pm 0.01 ; n=7)$. This difference is consistent with the reduced availability of $\mathrm{R} 669 \mathrm{H}$ channels in the "steady-state" protocol of Figure 5. As in the two-pulse protocol of Figure 6, data from the sequential protocol show a dramatic slowing for the recovery of the $I_{M}$ component of R669H (1600 $\pm 220 \mathrm{msec} ; n=11)$ compared with that of WT $(230 \pm 21 \mathrm{msec} ; n=7)$. The second exponential component of recovery, corresponding to $I_{S}$, was also slowed for $\mathrm{R} 669 \mathrm{H}(37 \pm 4.0$ sec compared with $15 \pm 0.7 \mathrm{sec}$ for WT), but the relative change was not as great as that observed for the $I_{M}$ component. During these very prolonged protocols, we noted a tendency for the peak amplitude of $I_{\mathrm{Na}}$ during recovery eventually to become slightly greater than the mean value measured for the reference pulses. This increase presumably represents further recovery of chronically inactivated channels, despite our waiting for at least $10 \mathrm{~min}$ at $-120 \mathrm{mV}$ before starting the sequential recovery protocol.

Composite data from all conditioning pulse durations revealed that wild-type channels relaxed from $I_{M}$ to the resting/available state with a time constant of $190 \pm 23 \mathrm{msec}(n=28)$ and from the slowly developing $\left(I_{S}\right)$ state with a relaxation time constant of $11 \pm$ $3.0 \mathrm{sec}(n=17)$. In comparison, $\mathrm{R} 669 \mathrm{H}$ channels exhibited relaxation time constants of $900 \pm 97 \mathrm{msec}(n=33)$ or approximately fivefold longer than that of wild type for the $I_{M}$ component $(p<$ $0.001)$ and $23 \pm 4.8 \mathrm{sec}(n=24)$ or approximately twofold longer than that of wild type $(p<0.005)$ for the $I_{S}$ component. There was no difference in the time constant for recovery of the fast component $(3.5 \pm 0.17 \mathrm{msec}$ for WT and $4.2 \pm 0.15 \mathrm{msec}$ for $\mathrm{R} 669 \mathrm{H})$. These estimates were obtained by simultaneously fitting the recovery data after the $40 \mathrm{msec}, 1 \mathrm{sec}, 60 \mathrm{sec}$, and $240 \mathrm{sec}$ conditioning pulses with a set of four triple-exponential curves that were constrained to have the same values for the time constants. The relative amplitude of each component varied with the conditioning pulse duration [for details, see Hayward et al. (1996)].

The excitability defect in HypoPP muscle occurs in association with hypokalemia (serum $\left[\mathrm{K}^{+}\right]$of $<2.5 \mathrm{~mm}$ ). We tested whether the gating abnormalities observed for $\mathrm{R} 669 \mathrm{H}$ were sensitive to extracellular $\left[\mathrm{K}^{+}\right]$. Reducing the bath $\left[\mathrm{K}^{+}\right]$from 4 to $1 \mathrm{~mm}$ did not alter the gating of $\mathrm{R} 669 \mathrm{H}$ or of WT channels. In R669H channels, recovery of the $I_{M}$ and $I_{S}$ components were still slowed fivefold and twofold, respectively (data not shown).

\section{Use-dependent inactivation during a pulse train}

Enhanced slow inactivation of $\mathrm{Na}$ channels has been shown to augment the use-dependent reduction of peak $I_{\mathrm{Na}}$ during prolonged trains of brief depolarizations (Wang and Wang, 1997), such as might occur in skeletal muscle during vigorous activity. We tested whether use-dependent inactivation of Na channels during a pulse train was enhanced in $\mathrm{R} 669 \mathrm{H}$ channels. Depolarizing pulses to $+10 \mathrm{mV}$ for $3 \mathrm{msec}$ were applied at 5 or $20 \mathrm{~Hz}$, from a holding potential of $-90 \mathrm{mV}$. Figure $8 A$ shows that a $5 \mathrm{~Hz}$ train applied for 2 min produced a modest degree of use dependence for WT channels (relative peak current, $0.80 \pm 0.03 ; n=5$ ), whereas a significantly greater fraction of the R669H channels became unavailable in response to the same train (relative peak current, $0.65 \pm 0.03 ; n=5)$. This difference was more pronounced with higher-frequency pulse trains. The use-dependent reduction of $I_{\mathrm{Na}}$ during a $20 \mathrm{~Hz}$ pulse train was two times greater for $\mathrm{R} 669 \mathrm{H}$ channels (relative residual current, $0.25 \pm 0.03 ; n=6$ ) than for WT ones (residual relative current, $0.53 \pm 0.04 ; n=6$ ).

\section{DISCUSSION}

We studied the gating properties of the skeletal muscle Na channel mutation $\mathrm{R} 669 \mathrm{H}$, a rare cause of hypokalemic periodic paralysis located at the outermost charged residue of the IIS4 voltage sensor. In contrast to $\mathrm{Na}^{+}$channel mutations associated with paralytic attacks in hyperkalemic periodic paralysis or paramyotonia congenita that disrupt fast inactivation and, in some cases, also disrupt

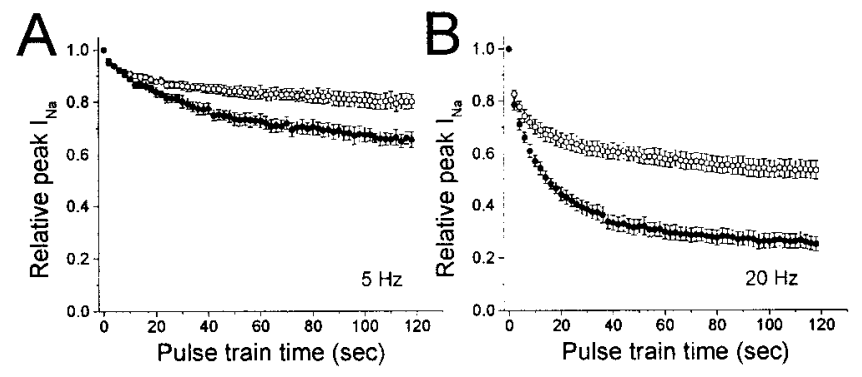

Figure 8. Use-dependent inactivation of $I_{\mathrm{Na}}$ during pulse trains was greater for $\mathrm{R} 669 \mathrm{H}$ than for wild-type channels. Trains of $3 \mathrm{msec}$ pulses to $+10 \mathrm{mV}$ from a holding potential of $-90 \mathrm{mV}$ were performed at $5 \mathrm{~Hz}(A)$ and $20 \mathrm{~Hz}(B)$. Peak $I_{\mathrm{Na}}$ for wild-type (open circles; $n=6$ ) and $\mathrm{R} 669 \mathrm{H}$ (closed circles; $n=6$ ) channels is plotted against the cumulative duration of the pulse train for every 10 th pulse $(A)$ or 40 th pulse $(B)$. Amplitudes were normalized to the value measured for the first pulse in the train.

slow inactivation, we found that R669H has negligible effects on fast gating but profoundly enhances slow inactivation. Slow inactivation was augmented in $\mathrm{R} 669 \mathrm{H}$ by an $\sim 10 \mathrm{mV}$ hyperpolarizing shift in voltage dependence, by more complete steady-state slow inactivation at depolarized potentials, and by a much slower rate of recovery. By using conditioning pulses of different lengths, we were able to demonstrate that the prolonged time course of $\mathrm{R} 669 \mathrm{H}$ recovery was caused by a fivefold to sevenfold slower recovery of the intermediate component of inactivation and a more modest (twofold) prolongation of recovery from the slow-inactivated state. The rate of entry to the intermediate- and slow-inactivated states was not significantly altered.

\section{Role of IIS4 in $\mathrm{Na}^{+}$channel gating}

The effects of the $\mathrm{R} 669 \mathrm{H}$ mutation on gating reported here are consistent with results of other mutagenesis studies performed on distal IIS4 residues in both skeletal muscle and other isoforms of the $\mathrm{Na}^{+}$channel. A charge-neutralizing arginine-to-cysteine substitution at the same position in hSkM1 (R669C) caused only a modest depolarizing shift of the $G-V$ relationship of $5-7 \mathrm{mV}$ (Mitrovic et al., 1998; Groome et al., 1999), similar to the small 3 $\mathrm{mV}$ rightward shift reported here. In the human cardiac $\mathrm{Na}$ channel isoform $\mathrm{hH} 1$, mutation of the outermost arginine in IIS4 to histidine caused a similar modest depolarizing shift in the conductance-voltage relationship and no change in the voltage dependence of inactivation (Chen et al., 1996). Studies of slow inactivation were not reported for the above mutations. Kontis and Goldin (1997) studied slow inactivation of the rat brain IIa $\mathrm{Na}$ channel in which the second outermost positive charge of IIS4 was neutralized by substitution with tryptophan. In agreement with our findings for $\mathrm{R} 669 \mathrm{H}$, the voltage dependence of slow inactivation was shifted to the left, although in their mutation the shift was $>10$ $\mathrm{mV}$ (Kontis and Goldin, 1997). This same study demonstrated that the voltage dependence of slow inactivation was preserved in a charge-conserving arginine-to-lysine missense mutation at the same position, supporting the idea that the effects on slow inactivation seen in our work result from changes in the charge density within IIS4. Histidine has a pK of 6-9, depending on its immediate environment; thus substitution of histidine for arginine is expected to result in at least a partial neutralization of the positive charge normally present at this position. However, we did not observe any change in fast- or slow-gating kinetics or voltage dependence when the external $\mathrm{pH}$ was varied between 6.5 and 8.0 for $\mathrm{R} 669 \mathrm{H} \mathrm{Na}$ channels (data not shown).

Several lines of experimental evidence have suggested that the S4 segments do not contribute equally to voltage-dependent gating in $\mathrm{Na}^{+}$channels. Mutations in IVS4 preferentially disrupt fast inactivation, without altering activation (Chahine et al., 1994; Chen et al., 1996). Using site-directed fluorescent labeling of each S4 segment, Cha et al. (1999) showed that changes in fluorescence signals from domains I and II voltage sensors follow activation and are not immobilized by fast inactivation, whereas signals from 
domains III and IV S4 segments tracked fast inactivation and its associated charge immobilization. Kontis and Goldin (1997) examined the effects of charge-neutralizing mutations in the second and fourth positively charged residues in each of the four S4 domains of the RBIIa $\mathrm{Na}^{+}$channel. Mutations in the second position of domains I and II, but not III or IV, caused pronounced shifts in the voltage dependence of slow inactivation, whereas only small changes were observed for activation or fast inactivation. Together with our findings of enhanced slow inactivation by $\mathrm{R} 669 \mathrm{H}$ in the first charged residue of IIS4, these data suggest that the S4 segment in domain II has a specialized role in regulating the voltage dependence of slow inactivation, particularly the charged residues at the first and second positions.

\section{Pathogenesis of hypokalemic periodic paralysis}

How enhanced slow inactivation caused by the R669H mutation results in the HypoPP phenotype is not immediately evident. Individuals with R669H-associated HypoPP have prolonged (hours to days) episodes of paralysis in conjunction with a reduction in serum potassium concentration, identical to the clinical presentation of HypoPP associated with L-type $\mathrm{Ca}^{2+}$ channel mutations (Bulman et al., 1999). The final common pathway in the development of weakness, for both $\mathrm{Ca}^{2+}$ and $\mathrm{Na}^{+}$channel-based HypoPP, is most likely a loss of excitability because of fiber depolarization and inactivation of $\mathrm{Na}^{+}$channels. In vitro recordings of HypoPP muscle fibers from patients with L-type $\mathrm{Ca}$ channel mutations demonstrate that sustained, aberrant depolarization of the sarcolemma forms the basis for these attacks of paralysis (Rüdel et al., 1984). HypoPP fibers depolarize when the bath $\left[\mathrm{K}^{+}\right]$is lowered from 4.0 to $2.5 \mathrm{~mm}$, whereas under the same conditions normal fibers hyperpolarize by several millivolts. The hypokalemiainduced depolarization in HypoPP fibers is not prevented by blockers of $\mathrm{Na}^{+}$(TTX) or L-type $\mathrm{Ca}^{2+}$ (nitrendipine) channels (Rüdel et al., 1984; Ruff, 1999). Microelectrode recordings of fibers from the hSkM1-R669H family have never been made, and consequently the resting potential of the affected fibers during attacks is unknown. It remains a possibility that changes in muscle membrane excitability in this family differ fundamentally from the more common L-type $\mathrm{Ca}^{2+}$ channel-associated HypoPP. Nevertheless, the clinical phenotype of hSkM1-R669H is sufficiently similar to the $\mathrm{Ca}^{2+}$ channel-linked disorder that it is unlikely that significant differences in basic pathophysiology exist.

Our results are surprising in view of recent proposals about the role of slow inactivation in diseases of muscle membrane excitability. A disruption of slow inactivation is thought to promote attacks of depolarization-induced weakness in HyperPP and PMC by impeding the ability of slow inactivation to attenuate aberrant persistent $\mathrm{Na}^{+}$currents arising from impaired fast inactivation or left-shifted activation (Hayward et al., 1999). In contrast, our results suggest that enhanced slow inactivation may contribute to, or at least does not preclude, the phenotype of prolonged weakness. Similarly, Bendahhou et al. (1999) reported recently a HyperPP-associated mutation, I1495F, in which prolonged weakness was a prominent component of the phenotype and enhanced slow inactivation was exhibited. The enhanced slow inactivation found in $\mathrm{R} 669 \mathrm{H}$ and $\mathrm{I} 1495 \mathrm{H}$ could result in a loss of muscle excitability. The availability of $\mathrm{Na}^{+}$channels at the resting potential would be decreased, and the decrease would be exacerbated by even a small depolarization of $V_{\text {rest }}$. Although this is a plausible mechanism for a loss of excitability, it does not explain how hypokalemia triggers attacks or why $V_{\text {rest }}$ is (presumably) depolarized during the attacks. We used trains of short depolarizations comparable with the duration of single action potentials and demonstrated that $\mathrm{R} 669 \mathrm{H}$ channels might become "trapped" in a slowinactivated state in a use-dependent manner over several minutes. This, too, may contribute to the exercise-induced loss of excitability and weakness.

Previous attempts to link functional defects mechanistically in HypoPP-associated mutant L-type $\mathrm{Ca}^{2+}$ channels to the electrophysiological changes in muscle during an attack have met with little success. A variety of alterations in the behavior of mutant L-type $\mathrm{Ca}^{2+}$ channels have been described. A common theme among this diversity is that external $\mathrm{Ca}^{2+}$ entry is predicted to be reduced in HypoPP muscle. $\mathrm{Ca}^{2+}$ current density is reduced in cultured human myotubes heterozygous for the R1239H (Sipos et al., 1995) or the R528H (Morrill et al., 1998) mutation. Heterologous expression studies of $\mathrm{R} 528 \mathrm{H}$ in mouse L cells (Lapie et al., 1996) or of R528H, R1239H, or R1239G channels in Xenopus oocytes (Morrill and Cannon, 1999) have also found a reduced current density for mutants compared with WT. Moreover, in oocytes we observed slowed activation for all three L-type channel mutants and accelerated deactivation for the R1239 mutant (Morrill and Cannon, 1999). The reduced current density and altered kinetics would act synergistically to reduce $\mathrm{Ca}^{2+}$ entry into $\mathrm{Hy}$ poPP muscle fibers. The link between altered $\mathrm{Ca}^{2+}$ entry and the episodic membrane depolarization and hypokalemia because of the trapping of $\mathrm{K}^{+}$in muscle remains to be discerned.

Several investigators have promoted the idea that secondary changes in ATP-regulated $\mathrm{K}^{+}$channel activity might be important in HypoPP attacks. Tricarico et al. (1999) found abnormal subconductance states and a reduced open probability of K-ATP channels in HypoPP fibers with the R528H L-type $\mathrm{Ca}^{2+}$ channel mutation. In another study of HypoPP fibers with the R528H mutation, Ruff (1999) showed that insulin potentiates the aberrant depolarization by reducing an inwardly rectifying $\mathrm{K}$ conductance. A decrement in the resting $\mathrm{K}^{+}$conductance is consistent with both hypokalemia because of the trapping of $\mathrm{K}^{+}$in the myoplasm and a depolarized shift in $V_{\text {rest }}$.

\section{Additional note}

Since the submission of this manuscript, Jurkat-Rott et al. (2000) have identified two additional missense mutations in an adjacent arginine (R672H and R672G) from five families with HypoPP phenotypes. Heterologous expression studies showed a reduced $\mathrm{Na}^{+}$current density and enhanced inactivation for both mutants. Thus our findings and those of Jurkat-Rott both imply that a reduced $\mathrm{Na}^{+}$current density contributes to the pathogenesis of HypoPP.

\section{REFERENCES}

Almers W, Stanfield PR, Stühmer W (1983) Slow changes in currents through sodium channels in frog muscle membrane. J Physiol (Lond) 339:253-271.

Bendahhou S, Cummins TR, Tawil R, Waxman SG, Ptacek LJ (1999) Activation and inactivation of the voltage-gated sodium channel: role of segment S5 revealed by a novel hyperkalaemic periodic paralysis mutation. J Neurosci 19:4762-4771.

Bulman DE, Scoggan KA, van Oene MD, Nicolle MW, Hahn AF, Tollar LL, Ebers GC (1999) A novel sodium channel mutation in a family with hypokalemic periodic paralysis. Neurology 53:1932-1936.

Cannon SC (1998) Ion channel defects in the hereditary myotonias and periodic paralyses. In: Molecular neurology (Martin JP, ed), pp 257-277. New York: Scientific American.

Cannon SC (2000) Spectrum of sodium channel disturbances in the nondystrophic myotonias and periodic paralyses. Kidney Int 57:772-779.

Cannon SC, Brown Jr RH, Corey DP (1993) Theoretical reconstruction of myotonia and paralysis caused by incomplete inactivation of sodium channels. Biophys J 65:270-288.

Cha A, Ruben PC, George ALJ, Fujimoto E, Bezanilla F (1999) Voltage sensors in domains III and IV, but not I and II, are immobilized by $\mathrm{Na}^{+}$ channel fast inactivation. Neuron 22:73-87.

Chahine M, George Jr AL, Zhou M, Ji S, Sun W, Barchi RL, Horn R (1994) Sodium channel mutations in paramyotonia congenita uncouple inactivation from activation. Neuron 12:281-294.

Chandler WK, Meves H (1970) Slow changes in membrane permeability and long-lasting action potentials in axons perfused with fluoride solutions. J Physiol (Lond) 211:707-728.

Chen LQ, Santarelli V, Horn R, Kallen RG (1996) A unique role for the S4 segment of domain 4 in the inactivation of sodium channels. J Gen Physiol 108:549-556.

Cummins TR, Sigworth FJ (1996) Impaired slow inactivation in mutant sodium channels. Biophys J 71:227-236.

Featherstone DE, Richmond JE, Ruben PC (1996) Interaction between fast and slow inactivation in SkM1 sodium channels. Biophys J 71:3098-3109.

Fontaine B, Vale-Santos J, Jurkat-Rott K, Reboul J, Plassart E, Rime CS, Elbaz A, Heine R, Guimaraes J, Weissenbach J, Baumann N, Fardeau M, Lehmann-Horn F (1994) Mapping of the hypokalaemic periodic 
paralysis (HypoPP) locus to chromosome 1q31-32 in three European families. Nat Genet 6:267-272.

George Jr AL, Komisarof J, Kallen RG, Barchi RL (1992) Primary structure of the adult human skeletal muscle voltage-dependent sodium channel. Ann Neurol 31:131-137.

Groome JR, Fujimoto E, George AL, Ruben PC (1999) Differential effects of homologous S4 mutations in human skeletal muscle sodium channels on deactivation gating from open and inactivated states. J Physiol (Lond) 516:687-698.

Hayward LJ, Brown Jr RH, Cannon SC (1996) Inactivation defects caused by myotonia-associated mutations in the sodium channel III-IV linker. J Gen Physiol 107:559-576.

Hayward LJ, Brown Jr RH, Cannon SC (1997) Slow inactivation differs among mutant $\mathrm{Na}$ channels associated with myotonia and periodic paralysis. Biophys J 72:1204-1219.

Hayward LJ, Sandoval GM, Cannon SC (1999) Defective slow inactivation of sodium channels contributes to familial periodic paralysis. Neurology 52:1447-1453.

Jurkat-Rott K, Lehmann-Horn F, Albaz A, Heine R, Gregg RG, Hogan K, Powers PA, Lapie P, Vale-Santos JE, Weissenback J, Fontaine B (1994) A calcium channel mutation causing hypokalemic periodic paralysis. Hum Mol Genet 3:1415-1419.

Jurkat-Rott K, Mitrovic N, Hang C, Kouzmekine A, Iaizzo P, Herzog J, Lerche H, Nicole S, Vale-Santos J, Chauveau D, Fontaine B, LehmannHorn F (2000) Voltage-sensor sodium channel mutations cause hypokalemic periodic paralysis type 2 by enhanced inactivation and reduced current. Proc Natl Acad Sci USA 97:9549-9554.

Jurman ME, Boland LM, Liu Y, Yellen G (1994) Visual identification of individual transfected cells for electrophysiology using antibody-coated beads. Biotechniques 17:874-881.

Kontis KJ, Goldin AL (1997) Sodium channel inactivation is altered by substitution of voltage sensor positive charges. J Gen Physiol 110:403-413.

Lapie P, Goudet C, Nargeot J, Fontaine B, Lory P (1996) Electrophysiological properties of the hypokalaemic periodic paralysis mutation $(\mathrm{R} 528 \mathrm{H})$ of the skeletal muscle alpha 1s subunit as expressed in mouse $\mathrm{L}$ cells. FEBS Lett 382:244-248.

McClatchey AI, Cannon SC, Slaugenhaupt SA, Gusella JF (1993) The cloning and expression of a sodium channel beta 1-subunit cDNA from human brain. Hum Mol Genet 2:745-749.
Mitrovic N, George Jr AL, Horn R (1998) Independent versus coupled inactivation in sodium channels. Role of the domain 2 S4 segment. J Gen Physiol 111:451-462.

Morrill JA, Cannon SC (1999) Effects of mutations causing hypokalaemic periodic paralysis on the skeletal muscle L-type $\mathrm{Ca} 2+$ channel expressed in Xenopus laevis oocytes. J Physiol (Lond) 520:321-336.

Morrill JA, Brown Jr RH, Cannon SC (1998) Gating of the L-type Ca channel in human skeletal myotubes: an activation defect caused by the hypokalemic periodic paralysis mutation R528H. J Neurosci 18:10320-10334.

Ptacek LJ, Tawil R, Griggs RC, Engel AG, Layzer RB, Kwiecinski H, McManis PG, Santiago L, Moore M, Fouad G, Bradley P, Leppert MF (1994) Dihydropyridine receptor mutations cause hypokalemic periodic paralysis. Cell 77:863-868.

Rüdel R, Lehmann-Horn F, Ricker K, Kuther G (1984) Hypokalemic periodic paralysis: in vitro investigation of muscle fiber membrane parameters. Muscle Nerve 7:110-120.

Rudy B (1978) Slow inactivation of the sodium conductance in squid giant axons. Pronase resistance. J Physiol (Lond) 283:1-21.

Ruff RL (1994) Slow Na+ channel inactivation must be disrupted to evoke prolonged depolarization-induced paralysis. Biophys J 66:542-545.

Ruff RL (1999) Insulin acts in hypokalemic periodic paralysis by reducing inward rectifier K+ current. Neurology 53:1556-1563.

Ruff RL, Simoncini L, Stuhmer W (1988) Slow sodium channel inactivation in mammalian muscle: a possible role in regulating excitability. Muscle Nerve 11:502-510.

Sipos I, Jurkat-Rott K, Harasztosi C, Fontaine B, Kovacs L, Melzer W, Lehmann-Horn F (1995) Skeletal muscle DHP receptor mutations alter calcium currents in human hypokalaemic periodic paralysis myotubes. J Physiol (Lond) 483:299-306.

Tricarico D, Capriulo R, Conte Camerino D (1999) Insulin modulation of ATP-sensitive $\mathrm{K}+$ channel of rat skeletal muscle is impaired in the hypokalaemic state. Pflügers Arch 437:235-240.

Vedantham V, Cannon S (1998) Slow inactivation does not affect movement of the fast inactivation gate in voltage-gated $\mathrm{Na}^{+}$channels. J Gen Physiol 111:83-93.

Wang SY, Wang GK (1997) A mutation in segment I-S6 alters slow inactivation of sodium channels. Biophys J 72:1633-1640. 